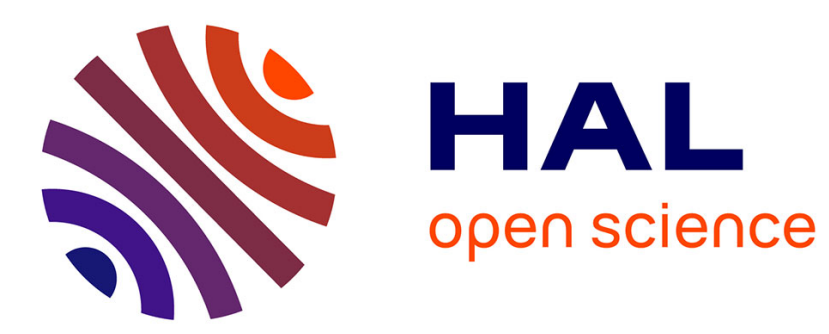

\title{
Self-oscillation and heat management in a LaFeSi based thermomagnetic generator
}

\author{
Smail Ahmim, Morgan Almanza, Vincent Loyau, Frédéric Mazaleyrat, \\ Alexandre Pasko, Fabien Parrain, Martino Lobue
}

\section{- To cite this version:}

Smail Ahmim, Morgan Almanza, Vincent Loyau, Frédéric Mazaleyrat, Alexandre Pasko, et al.. Selfoscillation and heat management in a LaFeSi based thermomagnetic generator. Journal of Magnetism and Magnetic Materials, 2021, 540, pp.168428. 10.1016/j.jmmm.2021.168428 . hal-03348584

\section{HAL Id: hal-03348584 \\ https://hal.science/hal-03348584}

Submitted on 16 Nov 2021

HAL is a multi-disciplinary open access archive for the deposit and dissemination of scientific research documents, whether they are published or not. The documents may come from teaching and research institutions in France or abroad, or from public or private research centers.
L'archive ouverte pluridisciplinaire HAL, est destinée au dépôt et à la diffusion de documents scientifiques de niveau recherche, publiés ou non, émanant des établissements d'enseignement et de recherche français ou étrangers, des laboratoires publics ou privés. 


\title{
Self-oscillation and heat management in a LaFeSi based thermomagnetic generator
}

\author{
Smail Ahmima ${ }^{\mathrm{a}}$, Morgan Almanza ${ }^{\mathrm{a}}$, Vincent Loyau ${ }^{\mathrm{a}}$, Frédéric Mazaleyrat ${ }^{\mathrm{a}}$, \\ Alexandre Pasko ${ }^{\mathrm{a}}$, Fabien Parrain ${ }^{\mathrm{b}}$, Martino LoBue ${ }^{\mathrm{a}}$ \\ ${ }^{a}$ Université Paris-Saclay, ENS Paris-Saclay, CNRS, SATIE, 91190, Gif-sur-Yvette, France \\ ${ }^{b}$ Université Paris-Saclay, CNRS, Centre de Nanosciences et de Nanotechnologies, 91120, \\ Palaiseau, France
}

\begin{abstract}
Harvesting and directly converting the largely untapped and omnipresent lowgrade heat into electricity has huge technological potential for supplying internet of things (IoT) devices, and wireless sensors in smart buildings applications. To this end, we present a self-actuating, autonomous, thermomagnetic generator (TMG) showing state-of-the-art net power output $4.2 \mu \mathrm{W}\left(240 \mu \mathrm{W} \mathrm{cm}{ }^{-3}\right)$, working over a $35.1{ }^{\circ} \mathrm{C}$ temperature difference. The core of our design is made of cheap and easily available piezoelectric buzzers and of commercial LaFeSi magnetocaloric materials from Vacumschmelze. The device self oscillating cycle is studied as a dynamical system with particular attention to the interplay between mechanical and thermal dynamics.
\end{abstract}

Keywords: thermomagnetic generator, thermal energy harvesting, magnetocaloric materials, self-oscillation, piezoelectric materials

\section{Introduction}

The number of Internet of Things (IoT) connected devices is expected to increase two-fold over the next five years [1] this will prompt a rising demand for autonomous power supply generators to sustain low-power wireless sensor nodes. Besides, the pressure to develop small, reliable, and compact energy scavenging devices to power up implantable medical electronics (IME) has been constantly rising for the past twenty years [2]. Considering the essential role played by wireless sensors nodes in the development of smart low carbon fingerprint buildings, and the growing relevance of IME to tackle endemic diseases and large scale health crisis, small scale energy harvesting can be counted between the key technological challenges of the next couple of decades.

Due to its ubiquity in buildings and urban environment, and in human body as well, low grade heat looks as one of the straightforward energy sources to

\footnotetext{
*Corresponding author

Email address: smail.ahmim@gmail.com (Smail Ahmim)
} 
target for applications whose aimed power-output falls in a range between some hundreds and tens of $\mu \mathrm{W}$ [1, 3].

Thermoelectric (TE) devices offer a direct conversion tool from a heat flux to electricity. Nevertheless keeping a constant temperature gradient, and avoiding detrimental heat leakages over small length scales, can be a difficult goal. This, jointly with the low factor of merit shown by available TE, represents a major shortcoming of this solution at the millimeter scale and for low grade heat sources [4].

For the last couple of decades, since the discovery of Giant Magnetocaloric effect [5], we witnessed the rising of a pretty large scientific community focused on caloric materials [6], and on their application to energy conversion [7, cooling, heat pumping, and power generation. A caloric material is a substance where the action of a field (viz. a thermodynamic intensive variable) induces a relevant response in one or both thermal state variables (i.e entropy and temperature). In general, the effect is magnified when the substance is on the verge of a ferroïc transition [6]. Caloric materials are named with a prefix pointing at the field triggering the effect so that magnetocaloric, electrocaloric, barocaloric, elastocaloric, refer to magnetic, electric, pressure, and stress fields respectively. In addition, multicaloric behavior may appear due to cross effects associated with coupling between order parameters.

Our focus on autonomy (i.e. battery-less devices) makes magntocaloric materials $(\mathrm{MCM})$ the more suitable choice due to the possibility to use magnets as field sources. Moreover magnetocaloric effect has been studied for a longer time, and we dispose of an unprecedented choice between families of materials that have been mostly optimized to be deployed in room temperature magnetic cooling devices [8]. An opportunity that can not be missed to give the old thermomagnetic generation (TMG) concept [9, 10, 11] a new chance.

Modifying the magnetic order through a temperature change is the core of TMG. The conversion to electrical energy is either done directly via Faraday law, or through an intermediate step through mechanical energy which is eventually converted by a transducer. Waste heat harvesting often imply an imposed temperature difference $\Delta T_{\text {res }}$ between the hot source and the heat sink, so that the power output as a function of $\Delta T_{\text {res }}$ is commonly used as a figure of merit. Some promising results have been obtained at $\Delta T_{\text {res }} \approx 100 \mathrm{~K}$. Recently, Gueltig and co-workers [4] realized a TMG showing an average electric power density of $118 \mathrm{mWcm}^{-3}$ for $\Delta T_{\text {res }}=140 \mathrm{~K}$. Before that, Chun et al. presented a TMG 12 with $38 \mathrm{mWcm}^{-3}$ for $\Delta T_{\text {res }}=80 \mathrm{~K}$.

Nonetheless, most of the waste heat available reservoirs are in the so called low grade form, involving a $\Delta T_{\text {res }}$ of at most few tens of degrees above room temperature. This motivates an increasing attention towards temperature spans within this interval. Working over $\Delta T_{\text {res }}=50 \mathrm{~K}$, Ujihara et al. 13. estimated a power of $3.6 \mathrm{~mW} / \mathrm{cm}^{2}$ (power per surface area of the active material). This power is indirectly extrapolated using the efficiency at resonance of a piezoelectric transducer. Guelting et al. [14] realized a TMG that shows an average power density of $1.6 \mu \mathrm{Wcm}^{-3}$ for $\Delta T_{\text {res }} \approx 25 \mathrm{~K}$. More recently, Waske et al. [15] show a maximum electric power density of $118 \mu \mathrm{Wcm}^{-3}$ with $\Delta T_{\text {res }}=30 \mathrm{~K}$. 
It is worth noting that the latter value, one of the higher published to date, represents the power output without considering the energy required to actuate the thermal switch (i.e. the pump keeping the flow of the fluid used to exchange heat).

Once thermal energy has been converted into magnetic one, conversion into electricity can be achieved through different methods (see [16] for a recent and exhaustive review). Direct conversion through Faraday law represents the most straightforward road [15]; in some cases an intermediate mechanical energy conversion step has been successfully used, jointly with Faraday law, in order to enhance conversion efficiency [14, 4, 17]. On the other hand the mechanical energy can also be harvested through other transducers as electroactive materials [12, and piezoelectric materials [13, 18].

Recently, we presented a piezoelectric based TMG [19] using a commercial first order MCM (CALORIVAC ${ }^{\circledR}$ from Vacumschmelze) as active substance. The adopted design is similar to the one presented in [13] with two major differences: the magnetic field source is a Halbach array in order to achieve high field gradients keeping demagnetizing fields as low as possible; the active substance undergoes a first order magnetic transition allowing higher efficiency at maximum power as shown in a recent study [20]. The thermal management is assured by alternatively moving the MCM from the hot source to the heat sink and vice versa, while the mechanical energy is recovered from the deformation of the beam where the material is fixed. This design, generally referred as linear [16, is particularly well suited to work over a well controlled thermodynamic four-strokes cycle (viz. two quick adiabats, and two heat exchange transformations) [21, and to reach a self-oscillating regime where no external actuation is needed to drive the device [22, 4, 19]. The device reaches up to $0.12 \mu \mathrm{W}$ electric power-output with a density of $6.8 \mu \mathrm{W} \cdot \mathrm{cm}^{-3}$ per volume of the MCM [19. It works over a temperature difference $\Delta T_{\text {res }}=38 \mathrm{~K}$, with a $0.8 \mathrm{~mm}$ linear displacement, at a frequency $f=0.12 \mathrm{~Hz}$. In spite of its state-of-the-art performance the main drawback of this design, and the key obstacle limiting its power output, is its relatively low working frequency.

Here we present a new prototype largely overcoming the performance of the previous one, with $4.2 \mu \mathrm{W}$ net power-output, $240 \mu \mathrm{W} \mathrm{cm}{ }^{-3}$ (i.e. over 30 times the output of the previous device), working over a similar temperature span, $\Delta T_{\text {res }}=35 \mathrm{~K}$, at an improved frequency, $f=0.41 \mathrm{~Hz}$.

In the following section we shall present and discuss the new design and its main advantages. We shall focus on the key objective of achieving a bistable dynamical cycle. The design and characterization of the new moving part will be described in detail and the directly measured output under optimal working conditions presented.

The third section is devoted to the study of the stability of the self-oscillating behavior, studied as a dynamical system driven by a conservative mechanical equation of motion coupled to a diffusive thermal equation. The dynamical system phase-space is sampled by varying an external parameter (i.e. the distance $d$ between the heat reservoirs), and its phase portrait discussed.

The physical mechanisms underlying the observed dynamics are discussed 
in the fourth section.

The relevance of this study is two-fold. On the one hand, it assesses the reliability of the device over different working conditions, a pretty relevant issue for an actual device. On the other, it allows to investigate the subtle interplay between the thermal and the mechanical cycles, showing that this is the key physical mechanism underlying the behavior and the performance of a TMG self-actuating device. A section devoted to discuss the main results and to the perspectives opened by the presented achievements will close the paper.

\section{Basic working principle and design}

A schematic representation of the prototype cross section and a set of pictures are shown in Fig. 1 and 2 respectively. The TMG is composed of a MCM plate, a disc spring, a field source, a hot source, and a heat sink. The MCM is a $\mathrm{La}(\mathrm{Fe}, \mathrm{Si}){ }_{13} \mathrm{H}$ plate $\left(0.25 \times 7 \times 10 \mathrm{~mm}^{3}\right)$ with a Curie temperature $T_{C}=30{ }^{\circ} \mathrm{C}$. The disc spring is made of two piezoelectric buzzers (see Fig. 2). They are inversely glued to each others through a thin $\mathrm{CuBe}$ ring. Each piezoelectric buzzer is cut, using a water-jet, in the shape of a cross in order to get the researched stiffness. The latter has been directly measured and is equal to $8.2 \mathrm{~N} . \mathrm{mm}^{-1}$. The total displacement of the MCM when switching from direct contact with one reservoir to the other is $d=0.48 \mathrm{~mm}$. The field source is an Halbach structure, built using $\mathrm{NdFeB}, 5 \times\left(1.5 \times 1.5 \times 10 \mathrm{~mm}^{3}\right)$ parallelepiped magnets. This configuration allows a stronger force with a steeper change over small displacements compared to the field generated by a single magnet [21]. The hot source temperature is kept constant through an electrical resistance while the cold reservoir is made with a water exchanger. An opening in the piezoelectric buzzer ensures the MCM direct thermal contact with the heat sink. The temperatures of the heat source and the of the heat sink are $T_{h}=51.5{ }^{\circ} \mathrm{C}$ and $T_{c}=16.4{ }^{\circ} \mathrm{C}$ respectively, giving a temperature difference between reservoirs $\Delta T_{\text {res }}=T_{h}-T_{c}=35.1^{\circ} \mathrm{C}$.

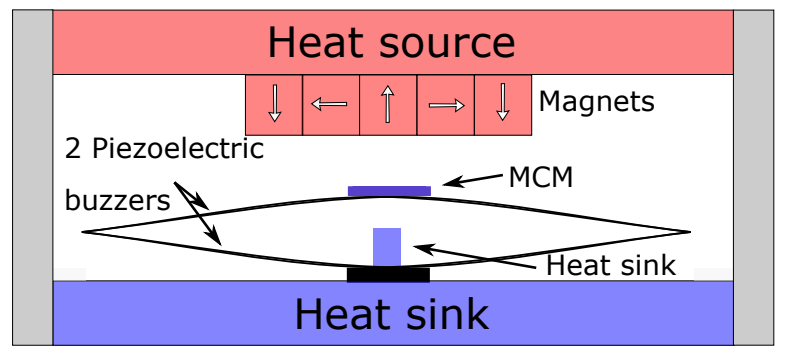

Figure 1: Schematic cross section of the TMG prototype.

The device works over a four strokes cycle driven by the competition between the magnetic and the elastic forces, $F_{m}$ and $F_{e}$ respectively. The latter has been measured, and depends linearly on the MCM position $x$ (viz. on the beam deformation) as shown in Fig. 4 (i.e. the green line passing trough points $A$ 


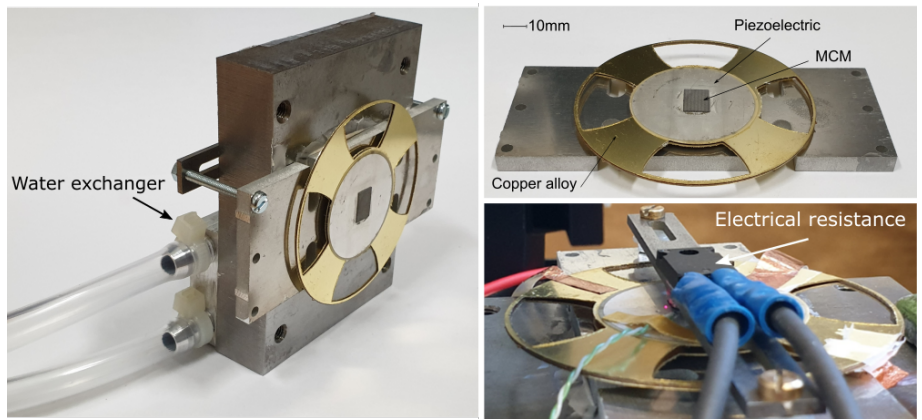

Figure 2: The beam (piezoelectric buzzer AB6505B from PUI Audio, Inc) and the MCM.

and $B$ ). The former depends on the field gradient (i.e. on the MCM position $x$ ), and on the MCM magnetization $M(T, H(x))$ where $T$ is the temperature of the material and $H(x)$ the magnetic field. Magnetic force isotherms computed with 3D Ansys MAXWELL, using as an input a set of $M(T, H)$ (see Fig. 3) curves measured with a VSM magnetometer are shown in Fig. 3 (see [19] for more details). Defects in the assembly with the magnets (misalignment) or in the magnets themselves lead to a lack-off flatness. This issue is addressed by introducing an equivalent air gap of $0.1 \mathrm{~mm}$ when the MCM is on the hot side.. The four steps composing the cycle can be described as follows:

Step 1: the MCM is kept in contact with the heat sink by the elastic force. It cools down until the ferromagnetic volume fraction makes $F_{m}$ overcoming $F_{e}$, namely when the material temperature corresponds to the magnetic force isotherm crossing the elastic force in the point $x_{1}=0.58 \mathrm{~mm}$ (i.e point $A$ in Fig. 4 matching the MCM position). This takes place at $T=18{ }^{\circ} \mathrm{C}$ (i.e. the yellow continuous line if Fig. (4).

Step 2: the MCM switches towards the hot source; if this step corresponds to a mechanical instability, the movement is expected to be fast enough to be considered an adiabatic transformation. It is worth noting that during the movement, due to the field change, the temperature will slightly increase due to the adiabatic temperature change.

Step 3: the MCM is in contact with the hot source, receiving heat and therefore becoming paramagnetic. During this process the magnetic force decreases till reaching the $F_{m}$ isotherm crossing $F_{e}$ at $x_{0}=0.1 \mathrm{~mm}$ (i.e. the $\mathrm{MCM}$ position). This takes place at $T=45{ }^{\circ} \mathrm{C}$ (point $B$ in Fig. 4).

Step 4: once reached the mechanical instability corresponding to point $B$ in Fig. 4 the MCM moves back to the heat sink. During this displacement the field decreases, triggering a negative adiabatic temperature change. This transformation too is expected to take place in adiabatic conditions.

Maximizing power by increasing frequency can be achieved through a reduction of the time associated with the four strokes. While the time spent to carry out steps 1 and 3 is related to the heat exchange process, steps 2 and 4 depend on the displacement velocity. On the one hand thermal contact is the 

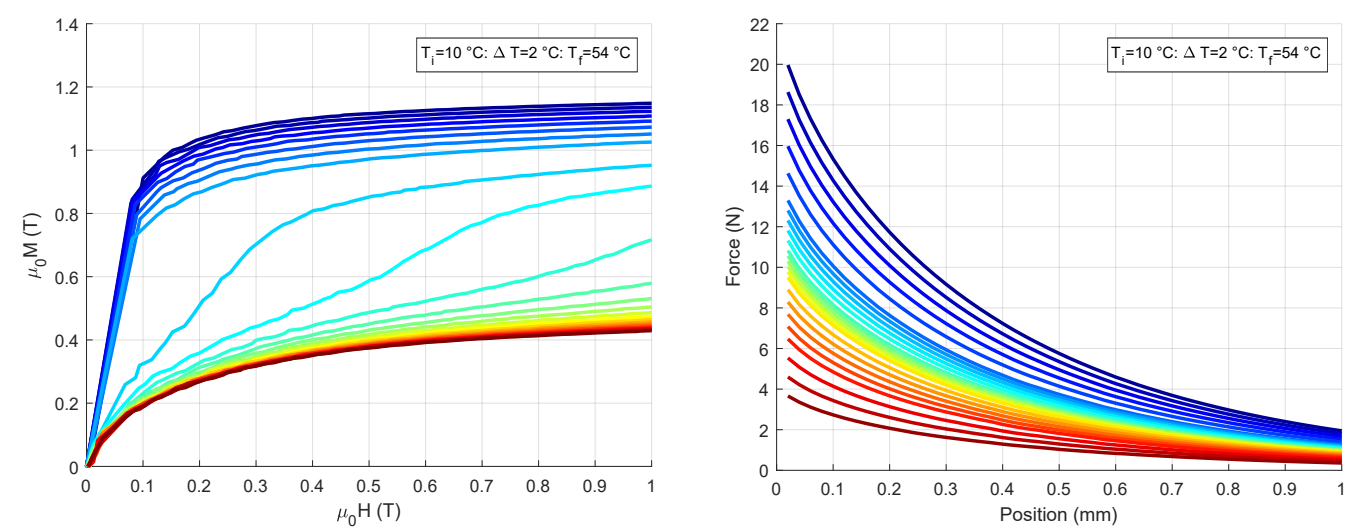

Figure 3: Left: $M(T, H)$ curves measured with a VSM magnetometer. Right: magnetic force isotherms computed with Ansys MAXWELL function of the air gap between the magnet and the MCM (i.e. the position of the MCM)

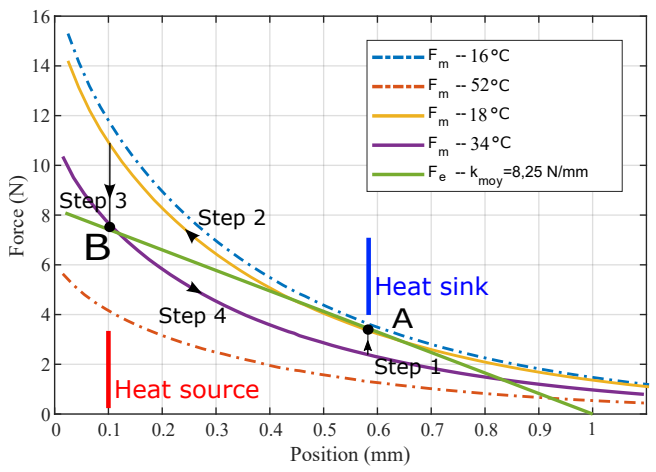

Figure 4: Magnetic force for different temperatures indicated in the legend and the elastic force of the disc spring function of the position. The MCM is in contact with the heat source (red) when the MCM is in $0.1 \mathrm{~mm}$ and with the cold source (blue) when the MCM is in $0.48 \mathrm{~mm}$. Points A and B are the cold and the hot switching points.

key to get fast heat exchange, on the other hand fast switching depends on a wise balance between forces in order to reach a bistable regime. In short, steps 2 and 4 must take place as instabilities with the driving force (magnetic one in step 2 and elastic one in step 4) largely overcoming the restoring one all along the displacement.

Given the magnetic force isotherms, the working cycle is determined by the slope of $F_{e}(x)$, where a fine beam-stiffness tuning is of the utmost relevance to get a bistable dynamical cycle. The disc spring has been designed to work within the elastic regime so that Von Mises stresses remain below $90 \mathrm{MPa}$ for copper and $50 \mathrm{MPa}$ for piezoelectrics.

After studying different configurations and shapes through FEM calculations 
with large deflection, we opted for a cross shaped beam made of two piezoelectric buzzers. A buzzer consists of a piezoelectric disk glued on the top of a metallic disk (a $\mathrm{CuBe}$ metallic alloy in this case) of larger diameter, as shown in frame $A$ of Fig. 5 . The stiffness of the $\mathrm{CuBe}$ disk has been tuned by cutting it in the cross-shape shown in frames $B$ and $C$ of Fig. 5 . The final setup presented here is obtained by assembling two buzzers in series (i.e. keeping the two piezoelectric disks on the outward sides).

A) One piezobuzzer

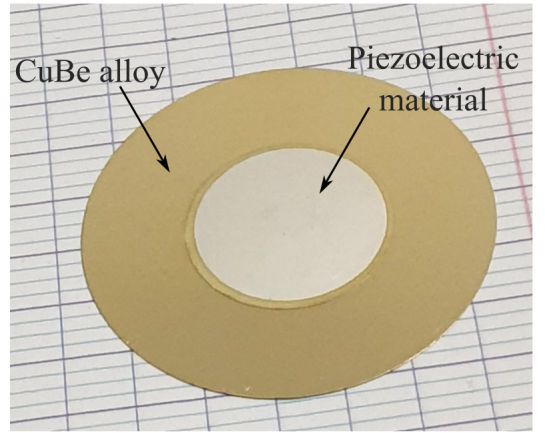

C) Assembly of the two cutted piezobuzzer

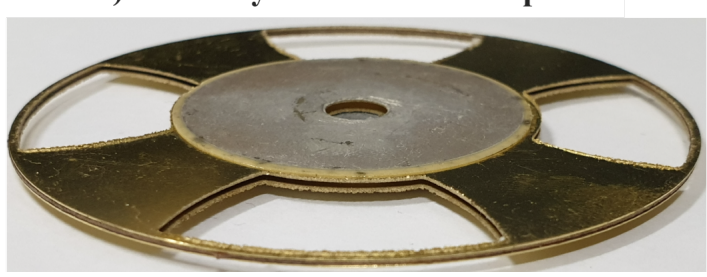

B) Cut of the two piezobuzzers

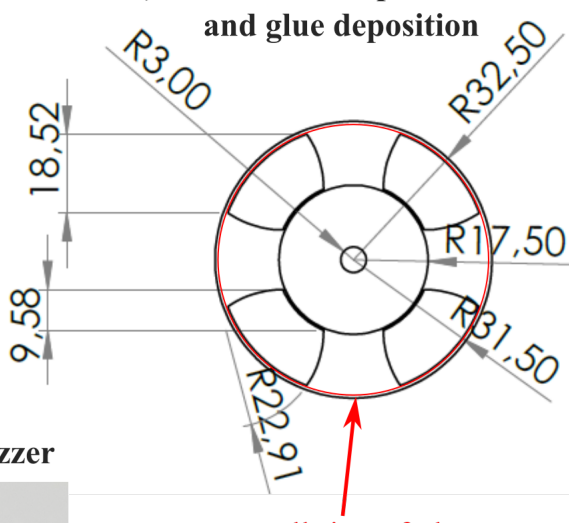

small ring of glue deposited on the edges

Figure 5: Assembly of the disc spring. A) a single buzzer with the larger CuBe disk and the piezoelectric disk; B) sizes and shape of the final cross cut; C) final setup with two cross-shape cut buzzers assembled in series.

The double disc spring reduces the device area, increasing the quantity of piezoelectric material and easing the assembly thanks to a mechanical response less sensitive to its fixation. Its stiffness as a function of the displacement has been directly measured, the result being the green line, shown in Fig.4 FEM simulations, shown in Fig 6 , confirm that the deformation is never getting beyond the elastic limit under a $d=1 \mathrm{~mm}$ displacement, more than twice the working point of the actual device. Considering the small working $\Delta T_{\text {res }}$, and the fact that the beam is never directly in contact with the reservoirs, a small but negligible temperature gradient through the bimorph is expected due to heat leakages from the MCM. Hereafter, no thermal effect is expected in the disc spring response.

The MCM displacement is measured using a laser sensor ILD1320 MICROEPSILON, the results are shown in Fig. 7. Steps 1 and 3 (i.e. MCM thermal contacts with the cold and hot end) last, $t_{c}=1.37 \mathrm{~s}$ and $t_{h}=0.9 \mathrm{~s}$ respectively. Steps 2 and 4 are steep, a signature of the researched bistable behavior. Working 


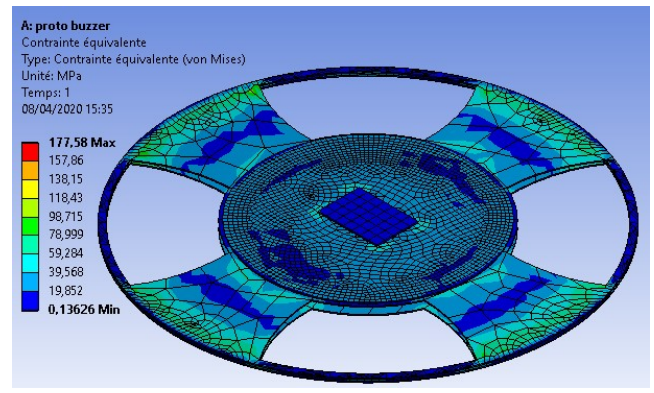

Figure 6: Finite element simulations of Von Mises stress on the different parts of the buzzer (copper and piezoelectric) for a displacement of the MCM $d=1 \mathrm{~mm}$.

over a total displacement $d=x_{1}-x_{0}=0.48 \mathrm{~mm}$ the device reach a frequency $f=0.41 \mathrm{~Hz}$ which is three times higher than the frequency of our previous prototype [19].

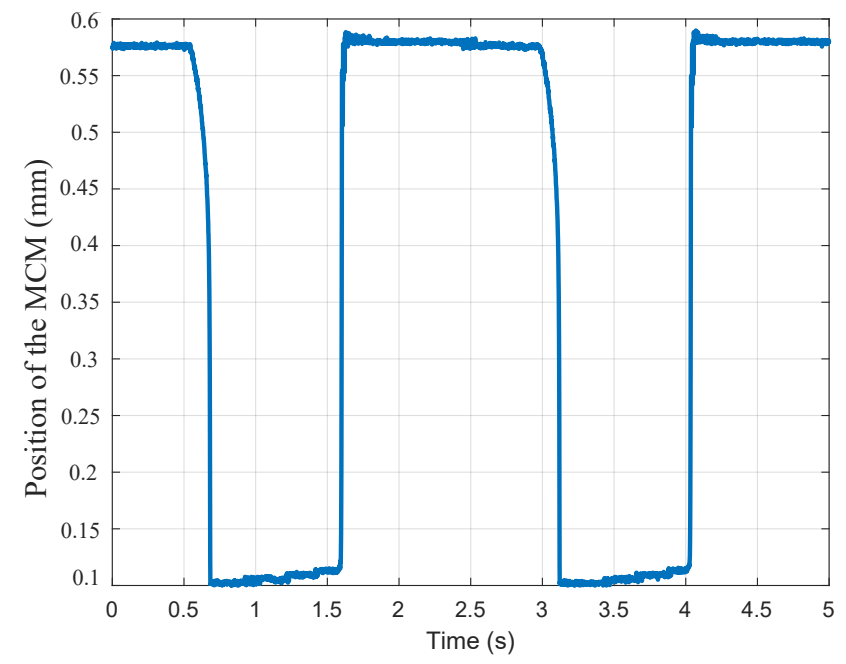

Figure 7: The position of the MCM as a function of time with a heat source and heat sink temperatures of $51.5{ }^{\circ} \mathrm{C}$ and $16.4{ }^{\circ} \mathrm{C}$ respectively (i.e. $\Delta T_{\text {res }}=35.1{ }^{\circ} \mathrm{C}$ ). The heat sink position $x_{1}=0.58 \mathrm{~mm}$, the heat source is $x_{0}=0.1 \mathrm{~mm}$.

The voltage induced on the piezoelectrics by the deformation is measured using a load resistor which maximizes the output power. Fig. 8 shows the voltage measured on the upper piezoelectric plate. The TMG net output electric power is $P_{\text {out }}=4.2 \mu \mathrm{W}$ (i.e. $240 \mu \mathrm{Wcm}^{-3}$ per volume of the active material) which corresponds to an electrical energy per cycle of $10.6 \mu \mathrm{J}$.

The power entering from the hot side is $1.7 \mathrm{~W}$, given the power output the efficiency is $210^{-6}$. The sources of efficiency reduction in our deign are 


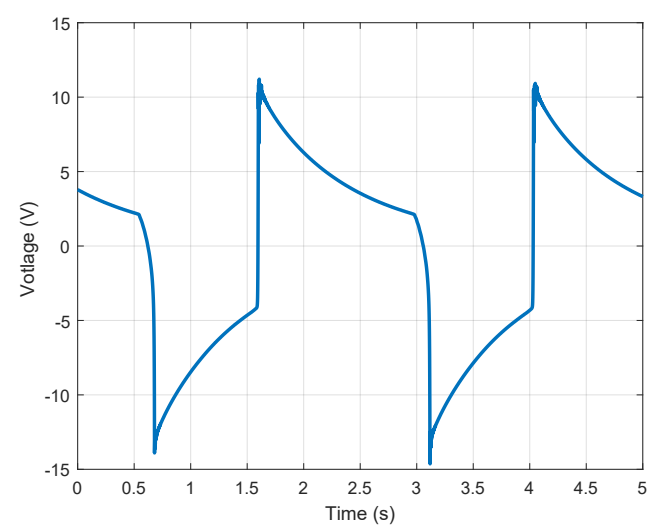

Figure 8: The voltage produced by the upper piezoelectric as a function of time.

manyfold. On the one hand only a small fraction of the incoming heat is actuallly passing through the TMG due to relevant heat leakages into air. Using the cited thermal model we estimate the global heat flux traversing the TMG to be $\sim 0.18 \mathrm{~W}$ (i.e. one order of magnitude lower than the actual incoming heat). Eventually, from our previous work, we estimate the final conversion efficiency into electric power to be reduced of a factor 1000. Indeed, harvesting low-grade thermal energy is, from the start, a matter of low efficiency, even Carnot's one being close to zero. Thence, here we put the main focus on autonomy (i.e. selfoscillation), and on reaching the power output needed by small MEMS scale systems.

\section{Study of working cycle as a dynamical system}

As discussed in the previous section, the device dynamical cycle is the result of the balance between the elastic force term $F_{e}(x)$, determined by the deformation (i.e. by the MCM position $x$ ), and the magnetic force $F_{m}(T, x)$. The latter depends on $x$ through the field gradient, and on both variables, $x, T$ through the magnetization $\mathbf{M}(T, H(x))$. To be sure, a pretty complex functional dependence the finite elements code has to deal with. A mayor issue here is that, beside the mechanical cycle whose variables are $x(t)$ and $\dot{x}(t)$, the material undergoes a thermodynamic (i.e. a thermal) cycle with a single dynamical variable $T(t)$ driven by a non-conservative equation of motion. This makes the global behavior a rather complex dynamical system operating on a three-dimensional phase-space whose points $\mathbf{x}(t) \equiv\{x, \dot{x}, T\}$, with $\mathbf{x} \in \mathbb{R}^{3}$, move along phase curves stemming from the coupling between the mechanical and the thermal dynamics. The phase velocity vector field is $\mathbf{v}(t) \equiv\{\dot{x}, \ddot{x}, \dot{T}\}$.

In what follows we shall see that this minimal model allows grasping, at least qualitatively, most of the relevant features we experimentally observed. A further refinement taking into account the piezoelectric material effect on the 
buzzer stiffens, associated with transducers electric tension, will be needed to explain some details of the MCM movement when the dynamics goes beyond the pure bistable regime. This introduces an additional functional dependence in the elastic force term, $F_{e} \equiv F_{m}(x, V(x))$, and a new variable, $V(t)$, making the phase-space a four dimensional one, with phase points $\mathbf{x}(t) \equiv\{x, \dot{x}, T, V\}$, and $\mathbf{x} \in \mathbb{R}^{4}$.

The dynamical system is constrained by two external parameters: the distance between the thermal baths $d=x_{1}-x_{0}$, limiting the displacement to the interval $x_{0} \leq x \leq x_{1}$; the temperature difference between the reservoirs, $\Delta T_{\text {res }}=T_{h}-T_{c}$. The latter drives the temperature dynamics through a Fourier thermal law,

$$
T \frac{d S(T, H(x))}{d t}=-K_{h}(x)\left(T-T_{h}\right)-K_{c}(x)\left(T-T_{c}\right)
$$

where $S$ is the entropy of the MCM, $T$ its temperature, $K_{h}(x)$, and $K_{c}(x)$ are the thermal conductance between the MCM and the hot and cold reservoir respectively, and, for the sake of simplicity, we neglect heat leakage through the beam. The dependence of $K_{h}$ and $K_{c}$ on the position $x$ describes heat conduction when the material is in contact with the bath (e.g. $K_{h}\left(x_{0}\right)$ represents the thermal conductivity between the hot reservoir and the MCM when they are in direct contact), as well as conduction through an air layer when the material lose contact with the heat baths (e.g. $K_{c}(x)$ drives the heat flux between the MCM and the cold bath through an air gap of thickness $x_{1}-x$, and $K_{h}(x)$ describe heat conduction between material and hot end through an air layer of thickness $x-x_{0}$ ).

In order to experimentally investigate the phase portrait of the TMG described as a dynamical system, we shall discuss its measured behavior by varying the distance $d$ between the reservoirs. This will allow to appreciate the complex interplay between mechanical and thermal variables in a self-oscillating TMG device based on an otherwise pretty simple design.

Hereafter, we shall study the device responses when changing the distance $d=x_{1}-x_{0}$ between reservoirs. The hot bath position is kept fixed at $x_{0}=0.1$ while the position of the cold reservoir, originally at $x_{1}=0.58 \mathrm{~mm}$ is changed either towards the positive or the negative direction increasing or decreasing $d$ respectively. More precisely, increasing or reducing $d$ will correspond to $x_{1}>$ $0.48 \mathrm{~mm}$ or $x_{1}<0.48 \mathrm{~mm}$ respectively. Measured displacement integral curves (i.e. $x(t)$ plot) corresponding to the latter case are shown in Fig. 9. Curves acquired after increasing $d$ are shown in Fig. 10.

The first issue apparent from the integral curves shown in Fig. 9 is that when the distance $d$ is reduced the system dynamics is still bistable (viz. switches between reservoirs are sharp, taking place over a negligible time scale), the total period increases (viz. frequency decreases), and the longer periods are mostly to be ascribed to the increase of the time the MCM stays in contact with the cold bath $t_{c}$. Table 1 allows a closer examination of the integral curves showing that while $t_{h}$ always increases when the gap between reservoirs is made thinner, the time spent in contact with the hot end $t_{h}$ undergoes an initial increase (i.e. 


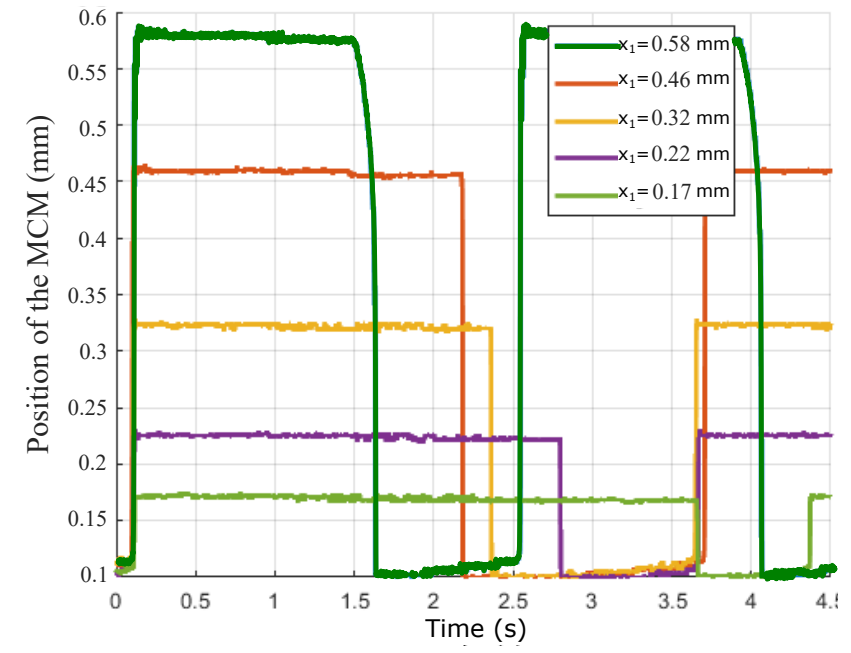

Figure 9: The displacement of the MCM (i.e. the $x(t)$ integral curves) for different values of the distance $d=x_{1}-x_{0}: x_{1}=0.58,0.46,0.32,0.22,0.17 \mathrm{~mm}$, and $x_{0}=0.1 \mathrm{~mm}$.

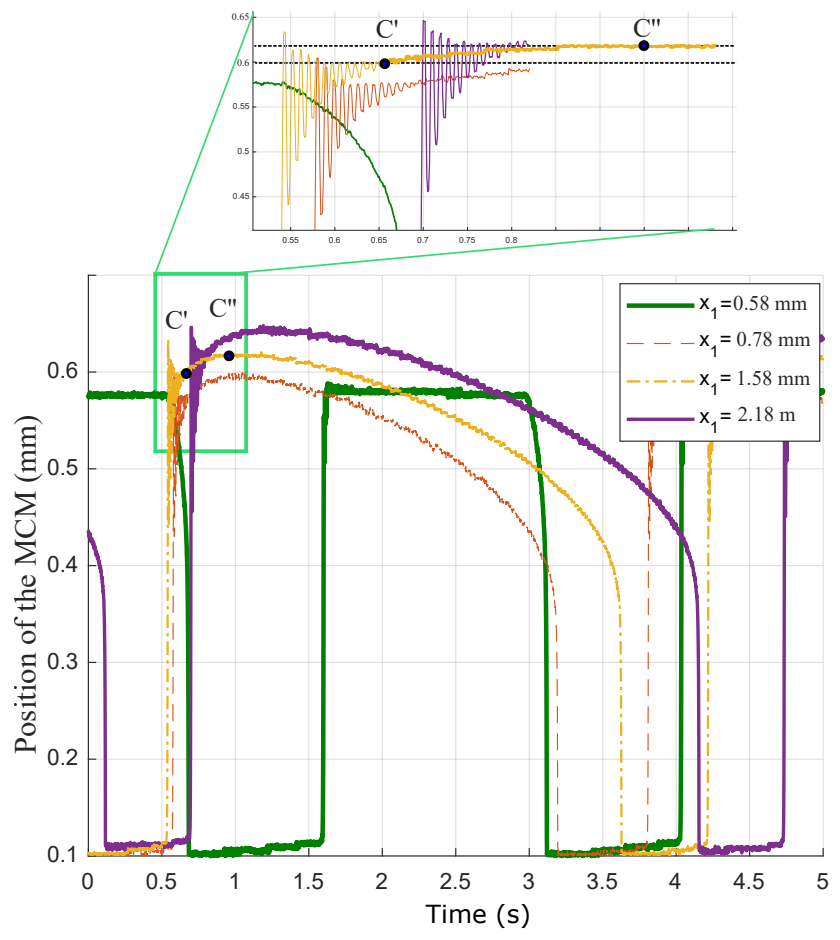

Figure 10: The displacement of the MCM for $x_{1}=0.58,0.78,1.58,2.18 \mathrm{~mm}$. From $x_{1} \geq$ $0.65 \mathrm{~mm}$, the MCM loses contact with the heat sink. 
Table 1: Data derived from Fig. 9

\begin{tabular}{|c|c|c|c|c|}
\hline$x_{1}(\mathrm{~mm})$ & Frequency $(\mathrm{Hz})$ & Period $(\mathrm{s})$ & $t_{h}(\mathrm{~s})$ & $t_{c}(\mathrm{~s})$ \\
\hline 0.58 & 0.41 & 2.44 & 0.9 & 1.37 \\
\hline 0.46 & 0.27 & 3.6 & 1.51 & 2 \\
\hline 0.32 & 0.28 & 3.53 & 1.27 & 2.24 \\
\hline 0.22 & 0.28 & 3.55 & 0.86 & 2.68 \\
\hline 0.17 & 0.23 & 4.25 & 0.7 & 3.54 \\
\hline
\end{tabular}

Table 2: Data derived from Fig. 10

\begin{tabular}{|c|c|c|c|c|}
\hline$x_{1}(\mathrm{~mm})$ & Frequency $(\mathrm{Hz})$ & Period $(\mathrm{s})$ & $t_{h}(\mathrm{~s})$ & $t_{c}(\mathrm{~s})$ \\
\hline 0.58 & 0.41 & 2.44 & 0.9 & 1.37 \\
\hline 0.78 & 0.31 & 3.24 & 0.61 & 2.41 \\
\hline 1.58 & 0.27 & 3.68 & 0.59 & 3.07 \\
\hline 2.18 & 0.25 & 4.04 & 0.56 & 3.46 \\
\hline
\end{tabular}

till $\left.x_{1}=0.46 \mathrm{~mm}\right)$, followed by a systematic reduction. On the other hand, Fig. 10 shows the integral curves when $x_{1}$ is increased (i.e. $x_{1}>0.58 \mathrm{~mm}$ ), and Tab 2 summarizes the relevant data relative to these curves.

Comparison between Fig. 9 and 10 makes clear that, decreasing the distance between baths, $x_{1}=0.58 \mathrm{~mm}$ represents the onset of the bistable dynamical regime. Actually, when $x_{1}>0.58 \mathrm{~mm}$ the system shows a complex behavior where the MCM no more attains contact with the cold end, and shows a damped oscillation over an intermediate equilibrium point followed by a transitory slow displacement ending when the material eventually switches back to the hot reservoir.

To understand this change in behavior as a function of $x_{1}$, it is worth using a phase portrait of the dynamical system. For the sake of simplicity we shall start from the minimal model where we neglect the role of the tension on the piezoelectric material so that, as stated above, phase points evolve in a three dimensional space, $\mathbf{x} \in \mathbb{R}^{3}$, with $\mathbf{x}(t)=\{x, \dot{x}, T\}$. In this case the phase portrait of the dynamical system in the plane $(x, T)$ is defined by the force-balance line (FBL) where the total force acting on the MCM is zero,

$$
F(x, T)=F_{e}(x)-F_{m}(T, x)=0 .
$$

The line, representing the sub-set of phase space where $\ddot{x}=0$ (i.e. with phase velocity $\left.\mathbf{v}_{F B L}=\{\dot{x}, 0, \dot{T}\}\right)$, splits the plane $(x, T)$ into two regions: one, below it, where the magnetic overwhelms the elastic force resulting in a total negative acceleration, dragging the MCM towards the hot end; the other, above it, where the elastic force is dominant and a positive force accelerates the material towards the heat sink. The point $C$, shown in Fig. 11, divides the FBL into a mechanical stable, and an mechanical unstable equilibrium branch.

The hot and cold end are shown as vertical continuous thick lines, red and blue respectively. This is to remind that they represent an external constraint 


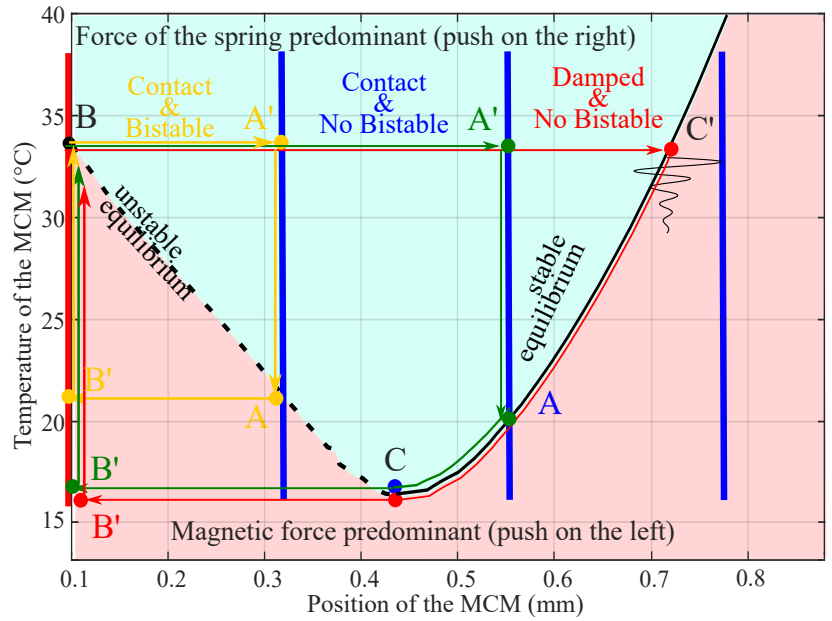

Figure 11: The FBL line, in black, defined by Eq. 2), divides the $(x, T)$ plane into two regions. One above the line where the elastic force is predominant. The other, below, where the magnetic force overwhelms the elastic one. The FBL line is divided into a stable (continuous black line), and an unstable (dotted black line) branch by the point $C$

to the dynamical system, in the form of two rigid impenetrable walls (i.e. a constraint fully described by the parameter $d=x_{1}-x_{0}$ ) limiting the phase space on the $x$ axis to the interval $x_{0} \leq x \leq x_{1}$.

Let us now discuss the typical phase trajectories associated with the integral displacement curves shown in Fig. 9 and 10.

Purely bistable cycles are described by the integral curves where $x_{1}<$ $0.58 \mathrm{~mm}$ shown in Fig9 As an example we can take the curve corresponding to $x_{1}=0.32 \mathrm{~mm}$ (the yellow line in Fig 9 ) where the MCM stays in direct contact with the reservoirs to heat up or cool down alternatively, and switches abruptly between them when reaching the temperature laying on the unstable branch of the force-balance line (corresponding on Fig 11 to point $B$ and the yellow point $\mathrm{A}$ ). If we neglect the adiabatic temperature change taking place when the material moves, corresponding to less than $2{ }^{\circ} \mathrm{C}$ given the material properties and the field change it is submitted to, the related phase trajectory can be represented as a rectangle in in $(x, T)$ plane. At $B^{\prime}$, where the system get in contact with the hot reservoir, the MCM can't move. However its temperature is lower than the temperature of the bath, the consequent heat exchange resulting in a temperature increase. More precisely, $\dot{x}=\ddot{x}=0$, and $\dot{T}>0$, with a phase-point velocity $\mathbf{v}=\{0,0, \dot{T}\}$ (represented in Fig 11 with an upward yellow arrow). Getting to point $B$, lying on the FBL, $\ddot{x} \geq 0$ and the MCM accelerates toward the heat sink. Neglecting heat exchanges with air (viz. adiabatic limit), and the adiabatic temperature change, the phase point moves along a horizontal line where $\dot{x}$, and $\ddot{x}$, are both positive, and $\dot{T}=0$, corresponding to a phase velocity vector $\mathbf{v}=\{\dot{x}, \ddot{x}, 0\}$ (horizontal leftward yellow arrow in Fig. 11). The displacement stops at $A^{\prime}$ where the material reaches the cold end (i.e. 
$\dot{x}=\ddot{x}=0$ ), and exchanges heat with the cold bath, here $\dot{T}<0$ as material temperature is greater than the cold bath one. Displacement form $A^{\prime}$ to $A$, where the vertical line crosses the FBL, takes place with $\dot{T}<0$ (the downward vertical yellow arrow in Fig 11 with phase velocity $\mathbf{v}=\{0,0, \dot{T}\}$. When the phase point reaches the force-balance line, at $A$, the MCM switch back to the hot end (horizontal leftward yellow arrow in Fig 11).

Let us now examine the integral curve corresponding to $x_{1}=0.58 \mathrm{~mm}$ (the green lines in Fig. 9 and 10 . While the switch from the hot to the cold bath is still sharp (i.e. fast material movement), the switch in the opposite sense takes place at a slower rate, at least down to $x \sim 0.42 \mathrm{~mm}$. This behavior can be explained tracing the corresponding phase trajectory on the $(x, T)$ plane as shown in Fig. 11. In this case the phase curve behaves in a pretty similar way to the purely bistable cycle along the $B^{\prime} B A^{\prime} A$ lines (green arrows in Fig 11 ). The main difference here is that the point $A$ lays on the stable branch of the FBL. This makes the system moving along the force-balance line, between $A$ and $C$, through a path of stable points. As far as the MCM loses direct contact with the cold reservoir, heat exchange continues, even if at a lower rate (i.e. $K_{c}(x)<K_{c}\left(x_{1}\right)$ when $\left.x \neq x_{1}\right)$, inducing a temperature decrease (i.e. $\dot{T}<0$ ), and a consequent displacement of the material (i.e. $\dot{x}<0$ ) depending on the FBL slope. This state of affairs suddenly changes when reaching $C$, where the system get unstable and switches towards the hot bath (represented by the green rightward arrow along $C B^{\prime}$ segment in Fig 11.

The third and more complex phase trajectory we shall consider corresponds to the behavior observed when $x_{1} \geq 0.58 \mathrm{~mm}$. Red lines and arrows in Fig. 11 describe the phase trajectory corresponding to the $x_{1}=0.78 \mathrm{~mm}$. In this case the MCM crosses the stable branch of the force-balance line at $x \sim 0.7 \mathrm{~mm}$, before getting to the heat sink. A damped oscillation lasting approximately $10^{-1} \mathrm{~s}$ takes place across the FBL till the phase point reaches the line at $C^{\prime}$. Afterwards the MCM is expected to moves along the FBL line exchanging heat with the heat sink through the air layer until reaching the point $C$ where the system becomes unstable and switches back to $B^{\prime}$. The damped oscillation can be explained in the frame of the minimal dynamical model by the change in potential energy associated with the material cooling down, and possibly by adding to the description energy losses associated with air viscosity and eddy currents. On the other hand, while the trajectory $C^{\prime} C$ shown in Fig 11 describes a slow MCM displacement towards the hot reservoir, quite similar to the one associated with the above described $A C$ path, it can hardly account of the MCM slow displacement towards the heat sink, just after the end of the oscillation, shown in Fig 10 between points $C^{\prime}$ and $C^{\prime \prime}$.

In order to explain this behavior, characterizing all the $x_{1}>0.58 \mathrm{~mm}$ integral curves, some substantial model improvements are required. The point here is that the minimal model neglects the effect of the piezoelectric material electric tension $V$ on the elastic force. Actually, when the MCM moves, deformation under charge of the piezoelectric materials imposes an additional recall force (i.e. always opposed to the displacement). This force relaxes over a scale time of seconds when the tension is discharged on a resistance (viz. the final energy 


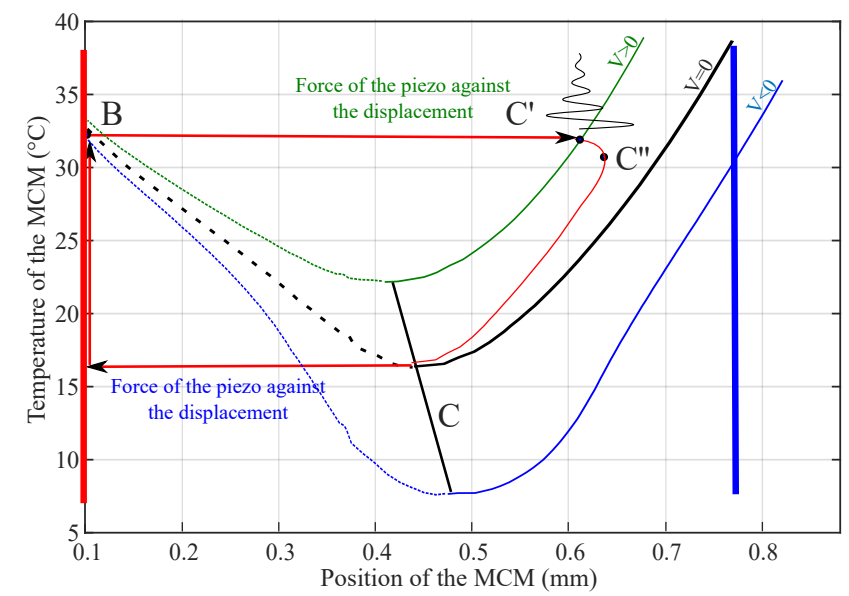

Figure 12: The force-balance line including the effect of piezoelectric tension on the elastic force. The path $C^{\prime} C^{\prime \prime}$ accounts of the observed slow displacement of the MCM towards the heat sink apparent in Fig 10

conversion step). To address this effect the elastic force must be described as a function of the position of the MCM $x$, of the tension on the piezoelectric transducers $V$, and of the sense of the deformation, namely $F_{e} \equiv F_{e}(x, V)$, the dynamical system phase space becoming a four dimensional one with $\mathbf{x} \equiv$ $\{x, \dot{x}, T, V\}$, and $\mathbf{x} \in \mathbb{R}^{4}$. Now the force-balance line in the plane $(x, T)$ changes its shape depending on different piezoelectric deformations and states of charge. FBL corresponding to different values of $x$ and $V$ are qualitatively represented in Fig 12 showing the way the $C^{\prime} C^{\prime \prime}$ path of Fig 10 is associated to the forcebalance line shape change during the piezoelectric tension discharge.

\section{Discussion}

This section shall address the different physical mechanism underlying the behaviors described above, and their role on the overall working performance of the device.

As maintaining a stable self oscillating regime, and getting high power output through frequency improvement are the main goals of this work, we shall focus on these two features.

Starting from self-oscillation, it is worth noting that in spite of its complexity, and of the non-linear, and non-conservative nature of most of its features, the dynamical system does not show chaotic behaviour when varying the parameter $x_{1}$ over the interval between $0.17 \mathrm{~mm}$ and $2.18 \mathrm{~mm}$, a pretty wide range indeed, spanning more than one order of magnitude.

Moreover, the self-oscillation is robust against variation of $d$. Namely, over the explored range of distances between reservoirs, the dynamical system is never getting into a fixed point stopping its oscillation. This second feature can be discussed more in detail through the projection of the phase portrait 
on the $(x, T)$ plane. A fixed point is a point of the phase space where all the components of the velocity vector are zero [23], within the minimal model this corresponds to $\mathbf{v}=\{\dot{x}, \ddot{x}, \dot{T}\}=0$. While $\ddot{x}=0$ is the mechanical equilibrium condition, $\dot{T}=0$ corresponds to thermal equilibrium. Equation 1 imposes:

$$
K_{h}(x)\left(T_{h}-T\right)-K_{c}(x)\left(T-T_{c}\right)=0 .
$$

This expression defines a boundary in Fig. 13, over the $(x, T)$ plane, getting from the point $\beta \equiv\left(0, T_{h}\right)$ to the point $\alpha \equiv\left(x_{1}, T_{c}\right)$, we shall refer to as the heat-balance line (HBL) (green line in Fig. 13).

Intersections points between the FBL and the HBL, are fixed points whenever they lay on the FBL stable branch (square points in Fig. 13). This case can be reached in two situations:

- when the MCM is in thermal equilibrium with one heat reservoir, a condition that can take place only if the material reaches the reservoir temperature (i.e. points $\alpha$ and $\beta$ ) before losing contact with its surface (i.e. before reaching the points $A$ and $B$ respectively), as shown in Fig. 13 (A);

- when the MCM is moving slowly enough to exchange heat with air (i.e. below the threshold speed where the displacement can be considered adiabatic), and the inward heat flux from the hot end equals the outward one towards the heat sink, as shown in Fig. 13.(B) on the A to C stable branch.

As the self-oscillation never gets jammed in the $d$ interval explored, we can infer that fixed points are absent from the phase space subset we sampled.

Summarizing, self-oscillation is rather robust against changes of the distance between reservoirs, and the dynamical systems never gets on the onset of chaotic behavior. Both these results can be ascribed to the stabilizing effect the heat flux through the air-gap holds over the dynamics. As a matter of fact, while non-linearity, damping, and forced oscillations are generally the causes of chaotic dynamics, a non-equilibrium heat flux, as the one described by Eq. (1), due to its trend towards a stationary maximum entropy production regime, stabilizes the dynamics making it less sensitive to changes in external parameters. Moreover it is noteworthy that in absence of the heat flux through air (viz. in vacuum), there wouldn't exist a HBL line and all the path along the FBL stable branch would be like a fixed points, this way, a huge part of the phase space unavailable to self-oscillating trajectories.

Focusing now on frequency, Table 1 and 2 show that the highest frequency matches with $x_{1}=0.58 \mathrm{~mm}$. On the one hand longer periods, more precisely, longer heat exchange times at the cold end $t_{c}$, are observed when $x_{1}$ is increased, as reported in Table 2. This is an expected result related to the fact that the $C^{\prime} C$ path of Fig 11 takes place farther away from the heat sink with the consequent $K_{c}(x)$ reduction. On the other hand, the frequency reduction when $x_{1}$ is reduced and the device is working in the purely bistable regime looks, at first sight, quite unreasonable. A further refinement of the thermal management model may help 

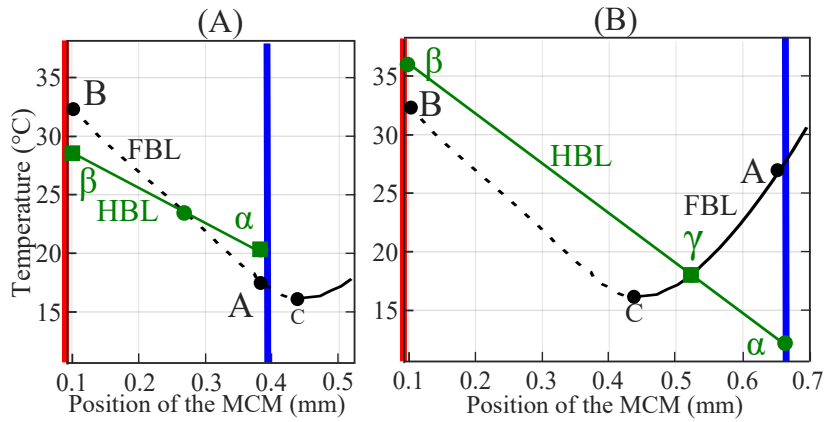

Figure 13: (A) the HBL is represented as a green line joining point $\beta$ to point $\alpha$. Here we show the case where $\beta$ lays below the FBL (below $B$ ) and $\alpha$ above the FBL (i.e. above $A$ ), so that both are fixed points of the dynamics (full green square point); (B) in this case the intersections between HBL and FBL with the reservoir surfaces are situated outside the phase-space subset accessible to the dynamics, on the contrary $\gamma$ (full green square point), representing the intersection between the HBL and the FBL stable branch, is a fixed point.

to gain an insight into this behavior. Actually, identifying the surface of the hot and cold end where the MCM gets in direct thermal contact with the reservoirs is somewhat oversimplifying the real situation. Any actual energy harvester is kept in thermal contact with reservoirs (e.g. the interior of a building and the outside atmosphere) through some thermal component showing a finite thermal conductance, say $K_{c}^{r}$ and $K_{h}^{r}$, referring to the cold and hot reservoir respectively. These heat exchange surfaces must exhibit a thermal capacity higher than the one of the active substance (i.e. the MCM in the present case) in order to keep their temperature constant all along the working cycle. However, the presence of a stationary heat flux through the device tends to reduce the temperature difference between the reservoir surfaces, the amount of the reduction depending on $K_{c}^{r}, K_{h}^{r}$, and on the flux intensity. From this point of view reducing the air gap between reservoir surfaces increases the heat flux intensity challenging the capacity of the heater, and of the cooler (i.e. the artificial heat reservoirs used in this setup) to keep $T_{h}$ and $T_{c}$ constant. Reducing $T_{h}$ and increasing $T_{c}$ means approaching the fixed point $\beta$ to point $B$ from above, and $\alpha$ to $A$ from below (see Fig. 13. This is slowing down heat transfer till stopping oscillation when $\alpha$, or $\beta$ eventually passes the points where the FBL intersect the reservoir surfaces. The difficulty to keep the temperature gradient against heat leakages is a rather general feature of thermal harvesting devices, particularly when working over small lengths scales, and with tiny temperature gradients (e.g. this is frequently addressed in the literature on thermo-electric generators [24]). Besides, the fact that the heat flux affects more the cold end than the hot one, as apparent from the disparity between the exchange time $t_{h}$ and $t_{c}$ apparent in Table 1 is something specific of our setup where the reduction of $d$ increases the distance between the cold surface and the water cooler so that while $K_{h}^{r}$ is unaffected, $K_{c}^{r}$ is proportional to $d$. This explanation can be easily checked by increasing the heat flux through $\Delta T_{\text {res }}$ change at fixed $d$. Within the above mentioned 
heat flux picture increasing $\Delta T_{\text {res }}$ is expected to modify the reservoirs surface temperature slowing down the oscillation and stopping it when a fixed point appears on one reservoir. This is what has been observed fixing the cold sink at $x_{1}=0.5 \mathrm{~mm}$ (viz. in the bistable region) and reducing the cooler temperature down to $T_{c}=11.1{ }^{\circ} \mathrm{C}$ (i.e. with $\Delta T_{r e s}=40.4{ }^{\circ} \mathrm{C}$ ) where the MCM eventually get stacked due to the appearance of a fixed point on the hot reservoir surface (i.e. the point $\beta$ temperature getting lower than point $B$ one as in Fig. 13.(A)).

\section{Conclusions}

Table 3 shows a summary of the data from the literature on the performance of existing TMG prototypes. Here we focus on two features, the power density normalized to the active material volume, and the working temperature difference between reservoirs $\Delta T_{\text {res }}$. Power densities span over four orders of magnitude, from tens of micro-Watts per cubic centimeter, up to more than one hundred milli-Watts per cubic centimeter.

However, ordering the data with respect to the working $\Delta T_{\text {res }}$ two distinct groups are apparent. Devices working over a temperature span of the order of $100{ }^{\circ} \mathrm{C}$ or more [12, 14, 4, 17] that generally get powers in the milli-Watts per cubic centimeter range, and prototypes working over a few tens degrees (i.e. $\leq 50{ }^{\circ} \mathrm{C}$ ) where the power density is in the range of tens or hundreds of micro-Watts per cubic centimeter.

In the first group the works on the prototype realized at the Karlsruhe Institute of Technology [14, 4, 17] deserve some special remarks. Firstly $\Delta T_{\text {res }}$ does not properly matches with the design presented there as, while the hot reservoir temperature is fixed, the heat sink one is varying during the fast oscillating cycle where the MCM exchanges heat with the disc spring and with air. Actually, besides reporting the best throughput published so far, these studies have the

soo great merit to show that frequency, and power improvements can be achieved in a system working under far-from-equilibrium thermodynamic conditions getting rid, with no regrets, of the comparison with Carnot cycle and its adiabats and isotherms. The main drawback here is that the optimal performance is reached at the price of using a rather hot heat reservoir, with $T_{h} \sim 170{ }^{\circ} \mathrm{C}$. The heat sink changes its temperature during the cycle but remains in the proximity of room temperature, this is the reason of the $\Delta T_{\text {res }} \gtrsim 100{ }^{\circ} \mathrm{C}$ reported in Table 3 .

In the second group, with $\Delta T_{\text {res }}$ below $50{ }^{\circ} \mathrm{C}$, a part for the theoretical power density upper bound reported in [13] (i.e. the actual output has not been published), the highest powers are shown by the TMG presented in [15] and by the prototype presented here, both working on the same temperature interval of about $30{ }^{\circ} \mathrm{C}$. Nonetheless it is worth noting that the device presented in [15] is not autonomous, needing a pump to actuate the heat-exchange fluid whose power consumption has not been reported, so that the net throughput, if any, is not known.

Summarizing, the thermo-magnetic harvester presented here show a state-ofthe-art performance and is, at the best of our knowledge, the sole autonomous 
Table 3: Comparison with different TMG prototypes

\begin{tabular}{|c|c|c|c|l|}
\hline & $\begin{array}{c}\text { Net power } \\
\left(\mu \mathrm{W} \mathrm{cm}^{-3}\right)\end{array}$ & $\begin{array}{c}\Delta T_{\text {res }} \\
\left({ }^{\circ} \mathrm{C}\right)\end{array}$ & Transducers & Comments \\
\hline$[15]$ & 320 & 30 & Coils & non-autonomous \\
\hline $\begin{array}{c}\text { This } \\
\text { work }\end{array}$ & 240 & 35.1 & Piezoelectric & measured \\
\hline$[19]$ & 6.8 & 38 & Piezoelectric & measured \\
\hline$[13$ & $28.3410^{3}$ & 50 & None & estimated \\
\hline$[12$ & $3810^{3}$ & 80 & PVDF & measured \\
\hline$[14$ & 1.6 & $\gtrsim 100$ & Coils & measured \\
\hline$[4]$ & $11810^{3}$ & $\gtrsim 100$ & Coils & measured \\
\hline$[17$ & $3310^{3}$ & $\gtrsim 100$ & Coils & measured \\
\hline
\end{tabular}

TMG prototype reported to show a net power output in the hundreds of microWatts per cubic centimeter, working in the vicinity of room temperature, and scavenging low grade heat over a range of few tens of degrees.

Furthermore, as a recent review on the application of caloric materials to energy conversion [16] puts it, the future of these technologies relies on frequency improvements that can hardly be achieved with devices needing a fluid to exchange heat with the reservoirs. In the design described here the MCM is the active material and the heat exchange substance at the same time, and the field source plays the two-fold role of triggering the caloric effect and switching the thermal management moving the material. In this context, the above presented study on the mechanical and thermal dynamics of the TMG, based on the phase portrait of an equivalent dynamical system, allows to grasp some key features of the device that would be otherwise difficult to appreciate.

On the one hand it does show how to take advantage of non-equilibrium fluxes, and further improvement towards a design recovering, through a mixed piezoelectric, and Faraday law transduction system, the energy associated with the damped oscillations observed in the $x_{1} \geq 0.58 \mathrm{~mm}$ case are envisaged. This would allow to avoid the impact with one or both reservoirs reducing undesired aging of the material, and recovering part of the kinetic energy, otherwise dissipated, whence an improvement of the overall efficiency.

On the other hand, the study makes clear that, in order to reduce the size of the system, getting towards a MEMS scale harvester, working under reduced air density (low grade vacuum) is an option to be seriously considered, not only because of the increasing role of air viscosity when the MCM thickens scales down, but because of the aforementioned effect of the heat leakage through air on the exchange surfaces.

Both options represent worthwhile opportunities in the research of novel solid-state based energy conversion technologies. 


\section{Acknowledgment}

This work benefited from the financial support of the project HiPerTherMag (ANR-18-CE05-0019) managed by the French National Research Agency. We would like to thank Dr. Alex Barcza from Vacuumschmelze for his kind support. Many thanks also to Eugene William from ENS Paris-Saclay and Nicolas Mercadié from IUT de Cachan for their priceless technical help.

\section{References}

[1] D. Newell, M. Duffy, Review of power conversion and energy management for low-power, low-voltage energy harvesting powered wireless sensors, IEEE Transactions on Power Electronics 34 (10) (2019) 9794-9805.

[2] B. Shi, Z. Li, Y. Fan, Implantable energy-harvesting devices, Advanced Materials 30 (44) (2018) 1801511.

[3] Z. Yi, F. Xie, Y. Tian, N. Li, X. Dong, Y. Ma, Y. Huang, Y. Hu, X. Xu, D. Qu, et al., A battery and leadless heart-worn pacemaker strategy, Advanced Functional Materials (2020) 2000477.

[4] M. Gueltig, F. Wendler, H. Ossmer, M. Ohtsuka, H. Miki, T. Takagi, M. Kohl, High-performance thermomagnetic generators based on heusler alloy films, Advanced energy materials 7 (5) (2017) 1601879.

[5] K. Pecharsky, Vitalij, A. Gschneidner Jr, Karl, Giant magnetocaloric effect in $\mathrm{Gd}_{5}\left(\mathrm{Si}_{2} \mathrm{Ge}_{2}\right)$, Physical Review Letters 78 (23) (1997) 4494.

[6] X. Moya, S. Kar-Narayan, D. Mathur, Neil, Caloric materials near ferroic phase transitions, Nature Materials 13 (5) (2014) 439-450.

[7] A. Kitanovski, U. Plaznik, U. Tomc, A. Poredoš, Present and future caloric refrigeration and heat-pump technologies, International Journal of Refrigeration 57 (2015) 288-298.

[8] J. Lyubina, Magnetocaloric materials for energy efficient cooling, Journal of Physics D: Applied Physics 50 (5) (2017) 053002.

[9] N. Tesla, Thermo-magnetic motor, uS Patent 396,121 (Jan. 15 1889).

[10] A. Edison, Thomas, Pyromagnetic motor, uS Patent 380,100 (Mar. 27 1888).

[11] L. Brillouin, P. Iskenderian, H., Thermomagnetic generator, Electrical Communication 25 (1948) 300.

[12] J. Chun, H.-C. Song, M.-G. Kang, H. B. Kang, R. A. Kishore, S. Priya, Thermo-magneto-electric generator arrays for active heat recovery system, Scientific Reports 7 (2017) 41383. 
[13] M. Ujihara, P. Carman, G., G. Lee, D., Thermal energy harvesting device using ferromagnetic materials, Applied Physics Letters 91 (9) (2007) 093508.

[14] M. Gueltig, H. Ossmer, M. Ohtsuka, H. Miki, K. Tsuchiya, T. Takagi, M. Kohl, High frequency thermal energy harvesting using magnetic shape memory films, Advanced Energy Materials 4 (17) (2014) 1400751.

[15] A. Waske, D. Dzekan, K. Sellschopp, D. Berger, A. Stork, K. Nielsch, S. Fähler, Energy harvesting near room temperature using a thermomagnetic generator with a pretzel-like magnetic flux topology, Nature Energy (2018) 1 .

[16] A. Kitanovski, Energy applications of magnetocaloric materials, Advanced Energy Materials 10 (10) (2020) 1903741.

[17] J. Joseph, M. Ohtsuka, H. Miki, M. Kohl, Upscaling of Thermomagnetic Generators Based on Heusler Alloy Films, Joule 4 (12) (2020) 2718-2732.

[18] L. Carlioz, Générateur piézoélectrique à déclenchement thermomagnétique, Ph.D. thesis, Institut National Polytechnique de GrenobleINPG (2009).

[19] S. Ahmim, M. Almanza, V. Loyau, A. Pasko, F. Mazaleyrat, M. LoBue, A thermal energy harvester using LaFeSi magnetocaloric materials, IEEE Transactions on Magnetics (2020) 1-1.

[20] M. Almanza, A. Pasko, F. Mazaleyrat, M. LoBue, First- versus secondorder magnetocaloric material for thermomagnetic energy conversion, IEEE

1. Transactions on Magnetics 53 (11) (2017) 1-6. doi:10.1109/TMAG. 2017. 2697398 .

[21] S. Ahmim, M. Almanza, A. Pasko, F. Mazaleyrat, M. Lobue, Thermal energy harvesting system based on magnetocaloric materials, The European Physical Journal Applied Physics 85 (1) (2019) 10902.

[22] A. Jenkins, Self-oscillation, Physics Reports 525 (2) (2013) 167-222.

[23] V. I. Arnold, Ordinary differential equations, MIT Press, Cambridge, Massachusetts, 1973.

[24] Y. Apertet, H. Ouerdane, O. Glavatskaya, C. Goupil, P. Lecoeur, Optimal working conditions for thermoelectric generators with realistic thermal coupling 97 (2) 28001. doi:10.1209/0295-5075/97/28001. 